PAPER

The influence of the entry region on the instability of a coflowing injector device

To cite this article: L Augello et al 2018 J. Phys.: Condens. Matter 30284003

View the article online for updates and enhancements.
Related content

Physics of liquid jets

Jens Eggers and Emmanuel Villermaux

- Dripping and jetting in microfluidic

multiphase flows applied to particle and

fibre synthesis

J K Nunes, S S H Tsai, J Wan et al.

- Microfluidic methods for generating

continuous droplet streams

G F Christopher and S L Anna

\section{Recent citations}

Dripping, jetting and tip streaming J M Montanero and A M Gañán-Calvo

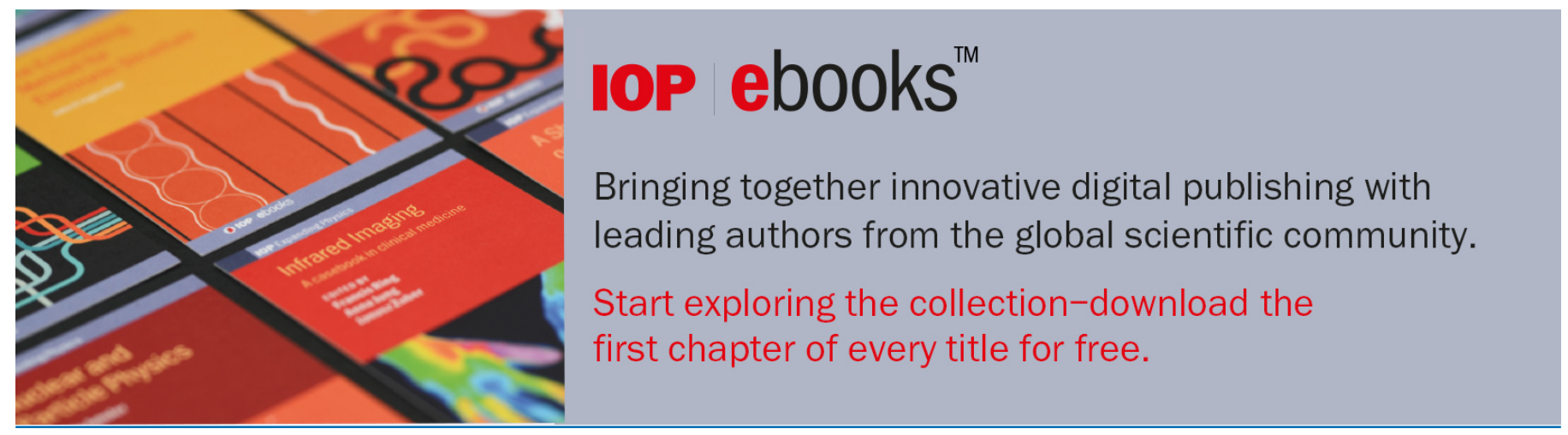

This content was downloaded from IP address 128.179 .130 .100 on 13/01/2021 at 15:30 


\title{
The influence of the entry region on the instability of a coflowing injector device
}

\author{
L Augello $\odot$, A Fani $\odot$ and F Gallaire \\ Laboratory of Fluid Mechanics and Instabilities, École Polytechnique Fédérale de Lausanne, Lausanne, \\ CH-1015, Switzerland \\ E-mail: francois.gallaire@epfl.ch
}

Received 30 January 2018, revised 17 May 2018

Accepted for publication 24 May 2018

Published 22 June 2018

\begin{abstract}
The occurrence of dripping and jetting regimes in a microfluidic coflowing injector device has been related recently to the spatio-temporal stability properties of the developed velocity profile. Dripping corresponds to an absolutely unstable flow while jetting is observed when the Rayleigh-Plateau instability of the core-annular jet is convective. In this work we take into account the effect of the entry region on the dripping to jetting transition by carrying out a global stability analysis of the steady two-phase base flow. We show that, depending on the flow parameters, the entry region can affect significantly the transition between the two regimes.
\end{abstract}

Keywords: Rayleigh-Plateau instability, coflowing jet, dripping/jetting

(Some figures may appear in colour only in the online journal)

\section{Introduction}

The production of droplets is of fundamental importance in industrial liquid-liquid contact processes, such as solvent extraction, ink jet printing, spray atomization, emulsification processes, and polymer extrusion (see [1, 2], to name a few). In microfluidic applications with two immiscible fluids, droplets are usually produced through passive techniques, where the flow field deforms the interface and promotes interfacial instabilities which lead to drop formation (see for instance [3] and the references therein).

One of the simplest possible devices consists of a co-axial injector, where the dispersed phase is injected in an outer carrier fluid which flows in a cylindrical tube. Cramer et al [4] carried out experiments on a co-flowing device with a needle placed inside a rectangular flow cell. They showed that the breakup of the liquid stream into droplets can be classified in two regimes: dripping, in which droplets pinch off near the injector tip, and jetting in which droplets pinch off from an extended thread downstream of the needle tip. The first regime is observed for small values of the inner phase flow rate $\tilde{Q}_{1}$, where the capillary force dominates. As $\tilde{Q}_{1}$ is increased (keeping the external phase flow rate $\tilde{Q}_{2}$ constant), viscous forces become comparable to the capillary ones and a transition to the jetting regime occurs. The transition depends also on the two fluids viscosities and on the interfacial tension.

Ten years ago, both Utada et al $[5,6]$ and Guillot and coworkers $[7,8]$ studied the stability of viscous jet confined within a viscous outer liquid in a microchannel. The latter carried out a local stability analysis of the developed flow profile (see figure 1(b)), herein also referred to as outlet profile, using a lubrication approximation and neglecting inertial terms. They interpreted the transition from dripping to jetting as a transition from an absolute to a convective instability, a concept widely applied in instabilities of shear flows and wakes (see [9] for details). In an absolutely unstable system perturbations can grow and withstand the mean advection, leading to self-sustained oscillations. In contrast, convectively unstable flows do not display intrinsic dynamics and essentially behave as amplifiers: external perturbations are amplified while propagating through the system. In co-axial devices an absolutely unstable configuration is related to a self sustained production of droplets (dripping), while a convectively unstable flow is expected to result in droplets which form at a finite distance downstream (jetting), only after the instability could grow sufficiently. The authors could identify a critical value of the capillary number $\mathrm{Ca}$ as a function of the flow parameters such as the fluid viscosities and the interface position $\left(\tilde{H}_{\text {out }}=H_{\text {out }} R\right.$ in figure $\left.1(\mathrm{~b})\right)$. 


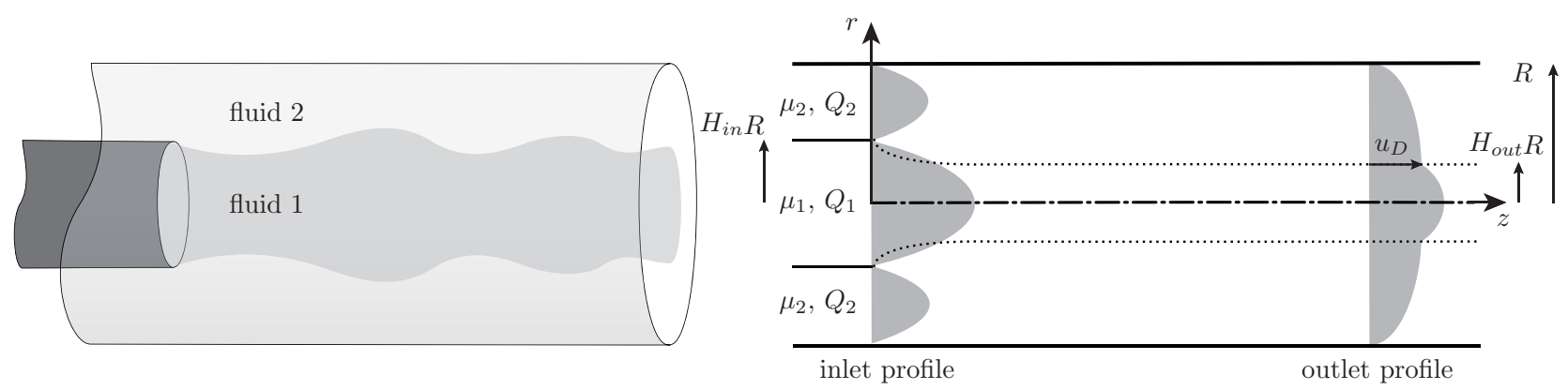

Figure 1. Flow domain and notations used in the text. On the left a 3D sketch of the core-annular flow, on the right a $2 \mathrm{D}$ view of the geometry. The dash-dotted line $(-\cdot)$ is the axisymmetric axis, the dotted line $(\cdot \cdot)$ is the interface, while the continuous line is the pipe wall at $r=R$. The inner fluid, with viscosity $\mu_{1}$ and flow rate $Q_{1}$, flows in an immiscible fluid with viscosity $\mu_{2}$ and flow rate $Q_{2}$.

A good agreement was found between the theoretical critical value and the capillary number at which transition from dripping to jetting was observed in experiments, confirming that the two transitions are related. The lubrication approach failed for low values of the jet radius $\tilde{H}_{\text {out }}$ : in that case the complete set of equations must be considered in order to have a correct agreement with experiments, as shown in $[10,11]$.

The local analysis carried out in these studies can be applied to parallel or slowly spatially evolving flows. Nonetheless, the flow is expected to vary rapidly from a bi-Poiseuille velocity profile close to the nozzle to the fully developed one as depicted in figure 1(b). In analogy with single phase pipe flow, the entry length is expected to scale like the pipe radius in the low Reynolds limit. In addition, the nozzle radius $\tilde{H}_{\text {in }}$ can be significantly different from the developed jet radius $\tilde{H}_{\text {out }}$, leading to an interface that varies along the stream-wise direction. To take into account non-parallel effects, a global stability analysis must be carried out. The studies using global methods have until now been concentrated on wakes behind solid obstacles and detached flows (see [12, 13] for reviews), but mainly of single phase flows. A global stability analysis using the long-wave Eggers and Dupont 1D approximation of a free jet (see [2] for a review of its many applications) was conducted in [14], who demonstrated the role of the axial curvature in the stabilization of a streched flow. Gordillo et al [15] studied the droplet production mechanisms of two coaxial streams in the so called tip-streaming regime, where the inner phase presents a cone-jet structure. They carried out a global stability analysis of the jet, by using a very elegant slenderbody approximation. The regime where uniformly sized droplets are produced was found to be associated to a globally stable flow, while the global instability of the cone-jet caused its unsteady oscillation and thereby produced a polydisperse spray. As far as the native $2 \mathrm{D}$ equations are concerned, a global stability analysis of the 2D wake of immiscible flows has been carried out in [16], who observed a counterintuitive destabilizing effect of the surface tension in this planar configuration. In the axisymmetric configuration, Cruz-Mazo et al [17] have recently conducted a global stability analysis of the flow-focussing geometry.

In this study we consider the flow in a co-axial injector and we investigate the effect of the entry region by means of a global stability analysis. The paper is organized as follows.
Section 2 describes the geometry, the governing equations and the numerical methods which are validated in section 3 . In section 4 we investigate the effect of fluid viscosities, flow rates and surface tension on the spatial evolution of the base flow. The stability properties of the base flow are then investigated by a global stability analysis of the $2 \mathrm{D}$ axisymmetric flow in section 5, before conclusions are drawn.

\section{Problem description}

We consider the flow of two incompressible and immiscible fluids in a pipe of radius $R$. One of the fluids is injected by using a nozzle of radius $\tilde{H}_{\text {in }}=H_{\text {in }} R$ as sketched in figure 1 . The two fluids have the same density $\rho$, which allows us to neglect gravity effects altogether, but different viscosity: $\mu_{1}$ for the inner fluid and $\mu_{2}$ for the outer one. The inlet velocity distributions are respectively a Hagen-Poiseuille profile with flow rate $\tilde{Q}_{1}=Q_{1} u_{D} R^{2}$ for the inner flow, and an annular profile with flow rate $\tilde{Q}_{2}=Q_{2} u_{D} R^{2}$ for the outer flow, where $u_{D}$ is the interface velocity reached by the jet once it has evolved downstream into the fully developed profile with interface location $H_{\text {out }} R$ (see section 2.1 for the explicit expressions of the velocity profiles). In the following, we neglect inertial effects compared to the capillary forces, $\rho u_{D}^{2} \ll \gamma / R$, where $\gamma$ is the surface tension. We have also checked a posteriori that the dimensionless eigenfrequencies of the unstable modes found in our analysis are of order one, implying that unsteady inertial terms scale like the convective inertial terms and can be neglected along with the latter.

The governing equations for the fluids are the incompressible Stokes equations in axisymmetric coordinates $(r, z)$, made dimensionless with the external pipe radius $R$, the fully developed velocity $u_{D}$ and the outer viscosity $\mu_{2}$. The problem is characterized by four dimensionless parameters:

$$
\mathrm{Ca}=\frac{\mu_{2} u_{D}}{\gamma}, \quad \lambda=\frac{\mu_{1}}{\mu_{2}}, \quad Q=\frac{Q_{1}}{Q_{2}}, \quad H_{\text {in }},
$$

where $\mathrm{Ca}$ is the capillary number, $\lambda$ the viscosity ratio, $Q$ is the flow rate ratio and $H_{\text {in }}$ the inlet interface location. All the flow quantities, such as the flow rates $Q_{1}$ and $Q_{2}$ and the developed interface position $H_{\text {out }}$, depend on the parameters introduced above (see for instance equation (6)). 


\subsection{Fully-developed solution and inlet velocity profiles}

The fully developed solution is well known as core-annular flow solution and can be expressed as follows, given the chosen non-dimensionalization

$$
\begin{aligned}
& u_{1}^{\mathrm{out}}(r)=1-\frac{1}{\lambda} \frac{H_{\mathrm{out}}^{2}-r^{2}}{H_{\mathrm{out}}^{2}-1} \text { for } 0 \leqslant r \leqslant H_{\mathrm{out}} \\
& u_{2}^{\mathrm{out}}(r)=1-\frac{H_{\mathrm{out}}^{2}-r^{2}}{H_{\mathrm{out}}^{2}-1} \text { for } H_{\mathrm{out}} \leqslant r \leqslant 1 .
\end{aligned}
$$

This piece-wise velocity profile both satisfies continuity of velocity (with nondimensional interface velocity 1 ) and continuity of shear stress. The resulting flow rates write

$$
\begin{aligned}
Q_{1} & =\pi\left(H_{\mathrm{out}}^{2}-\frac{H_{\mathrm{out}}^{4}}{2 \lambda\left(H_{\mathrm{out}}^{2}-1\right)}\right), \\
Q_{2} & =\frac{\pi\left(1-H_{\mathrm{out}}^{2}\right)}{2}, \\
Q & =\frac{2 H_{\mathrm{out}}^{2}}{\left(1-H_{\mathrm{out}}^{2}\right)}+\frac{H_{\mathrm{out}}^{4}}{\lambda\left(1-H_{\mathrm{out}}^{2}\right)^{2}} .
\end{aligned}
$$

To ensure conservation of flux, the following velocity profiles, ensuring null velocity on $r=H_{\text {in }}$ are imposed at the inlet

$$
\begin{gathered}
u_{1}^{\mathrm{in}}(r)=\frac{2 Q_{1}\left(H_{\mathrm{in}}^{2}-r^{2}\right)}{\pi H_{\mathrm{in}}^{4}} \text { for } 0 \leqslant r \leqslant H_{\text {in }}, \\
u_{2}^{\text {in }}(r)=\frac{2 Q_{2}\left(\ln \left(H_{\mathrm{in}}\right)\left(r^{2}-H_{\mathrm{in}}^{2}\right) /\left(1-H_{\mathrm{in}}^{2}\right)+\ln \left(\frac{r}{H_{\mathrm{in}}}\right)\right)}{\pi\left(\ln \left(H_{\mathrm{in}}\right)\left(1-H_{\mathrm{in}}^{2}\right)-2 \ln \left(H_{\mathrm{in}}\right)-\left(1-H_{\mathrm{in}}^{2}\right)\right)} \text { for } H_{\text {in }} \leqslant r \leqslant 1 .
\end{gathered}
$$

\subsection{Non-parallel steady solution}

In order to carry out a stability analysis, we first need to compute a steady solution. The base flow is computed assuming an axisymmetric solution by solving a single Stokes equation in the full domain coupled with the level set function that allows one to distinguish the two fluids (see $[18,19]$ for details on the method). The varying fluid properties, like for instance the viscosity, can be expressed as function of the level-set function. The advantage of this method is that we can perform numerical simulations with low capillary number $\mathrm{Ca}$ without having to parametrize the surface curvature (figure 2(a)). The governing equations become:

$0=\nabla \cdot\left[-\bar{p} \mathbb{I}+\mu(\phi)\left(\nabla \overline{\mathbf{u}}+(\nabla \overline{\mathbf{u}})^{T}\right)\right]+\mathrm{Ca}^{-1} \overline{\mathcal{C}} \overline{\mathbf{n}} \delta_{e}(\phi)$,

$\overline{\mathbf{u}} \cdot \nabla \phi=\nabla \cdot(D \nabla \phi)$,

$\nabla \cdot \overline{\mathbf{u}}=0$

where $\mu(\phi)$ denotes the viscosity, which is a function of the level set function $\phi$ :

$$
\mu(\phi)=1+(\lambda-1) \mathcal{H}_{e}(\phi),
$$

where $\mathcal{H}_{e}(\phi)$ is a regularized Heaviside function with continuous second derivative and transition thickness $e$ which depends on the mesh size. The capillary effects are modelled by the last term on the right-hand side of equation (9). The capillary force is localized at the interface by the regularized Dirac delta function $\delta_{e}(\phi)$ which is concentrated where $\phi=0$. The geometric properties of the interface are easily determined from the level-set function. The unit normal vector $\overline{\mathbf{n}}$ and the surface curvature $\overline{\mathcal{C}}$ are respectively given by

$$
\begin{gathered}
\overline{\mathbf{n}}=\frac{\nabla \phi}{|\nabla \phi|}, \\
\overline{\mathcal{C}}=-\nabla \cdot \overline{\mathbf{n}} .
\end{gathered}
$$

An important aspect of the level-set method is the treatment of the artificial diffusion $D$ required for numerical stability. In our model we used a streamline diffusion (streamline upwind Petrow-Galerkin method with tuning parameter $\delta_{\text {sd }}=0.25$ [20]). Finally, Dirichlet boundary conditions are used for $\overline{\mathbf{u}}$ and $\phi$ at the inlet and on the channel wall, while symmetry conditions are prescribed on the axis. At the outlet, the radial velocity is set to zero while the axial velocity and the level-set function both satisfy a Neuman condition.

The base flow simulations were carried out with COMSOL Multiphysics 4.2 a with $P 2-P 1$ discretization for the fluid and a $P 3$ discretization for $\phi$. The non linear base state is obtained with a Newton method where the linear systems are solved with the direct solver PARDISO [21].

The base flow computation, based on the level set method, is validated against results obtained with two other different numerical approaches. The first method used is the boundary element method (BEM), a so-called meshless interface tracking method where only the boundaries are discretized. Details on the method can be found in [22]. In addition, the base flow is also obtained by using a volume of fluid (VOF), along with a finite volume spatial discretization, implemented in the open source code Gerris Flow Solver [23]. Since these two methods solve for time-evolutions, they can be only compared in the $\mathrm{Ca}=\infty$ limit, in order to avoid RayleighPlateau like instabilities. Figure 3 shows the interface location obtained with the three methods for a flow with the following parameters: inner pipe radius $H_{\text {in }}=0.7$, viscosity ratio $\lambda=5$, flow ratio $Q=0.388$. We can see that the level set method is in remarkable agreement with the other two numerical techniques. For the base flow calculation, the level set approach is preferred because the Newton method also allows one to find steady unstable solutions. As already mentioned, both the BEM and the VOF unsteady codes used here are indeed prompt to reflect the instability of the liquid jet.

\subsection{Global stability analysis}

The stability properties of a steady base-flow can be assessed through a linear stability analysis by considering the time evolution of a small perturbation sought in term of a normal mode. Nonetheless the linearization of the level set formulation is particularly challenging due to the presence of the 
Single domain

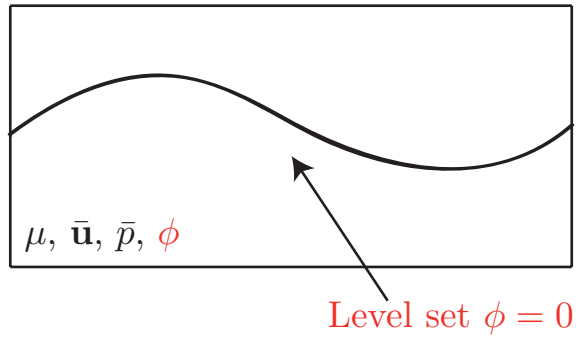

(a)

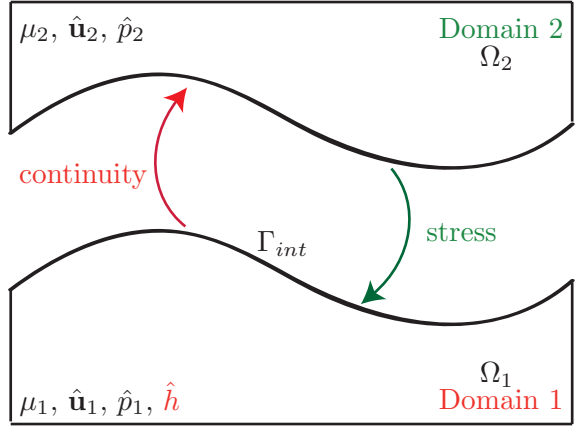

(b)

Figure 2. Base flow (a): the two fluid are computed in the same fixed grid by implementing the Stokes equation of a single velocity field in the full domain and the two layers are distinguished by a presence of the level-set function. Perturbed flow (b): the two fluid are computed in two different grid by implementing the Stokes equations of two velocity fields with the addition of the linearized boundary conditions at the interface. (a) Base flow. (b) Global stability.

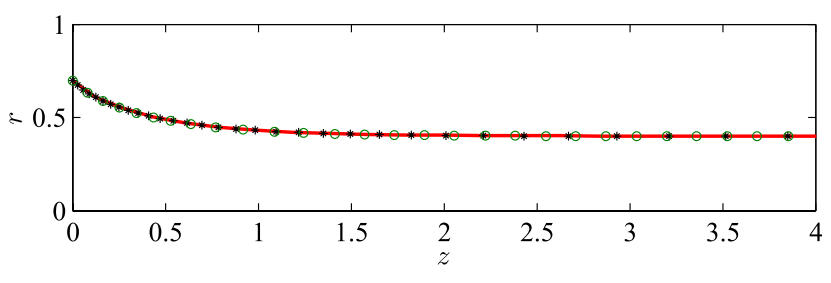

Figure 3. Comparison of the interface position found with the level-set method (red full line), boundary element method (* marker) and VOF description (o marker) for a co-axial flow. Simulations done for $H_{\text {in }}=0.7, Q_{1}=0.512, Q_{2}=1.319$, $Q=0.388, \lambda=5$ and $\mathrm{Ca}=\infty$. The fully developed interface location is $H_{\text {out }}=0.4$.

Dirac delta function. In this study, we preferred to use an alternative approach for the stability problem formulation. The problem is formulated in two different grids (one for each fluid) that can interact through the boundary conditions at the interface, i.e. continuity of the velocity and stress jump (figure 2(b) and equations (19) and (18)). The flow variables are the velocity $\mathbf{U}_{i}=u_{i} \mathbf{e}_{z}+v_{i} \mathbf{e}_{r}$ and the pressure $P_{i}$ where the index $i=\{1,2\}$ denotes respectively the inner and the outer flow. In addition, the interface $\Gamma_{\text {int }}$ is parametrized by a function $H(z, t)$ where the associated scalar field $F(r, z, t)=r-H(z, t)$ vanishes $F(H, z, t)=0$. The nonlinear governing equations for the two fluids are:

$$
\begin{aligned}
0 & =\nabla \cdot \sigma_{\mathrm{i}} \\
\nabla \cdot \mathbf{U}_{i} & =0 \\
\partial_{t} F & =-\mathbf{U}_{\mathrm{i}} \nabla F
\end{aligned}
$$

where $\sigma_{i}=-P_{i} \mathbb{I}+\mu_{i}\left(\nabla \mathbf{U}_{i}+\nabla \mathbf{U}_{i}^{T}\right)$ is the stress tensor. Note that $\mu_{i}$ here is $\lambda$ for $i=1$ and 1 for $i=2$. Equation (17) is the kinematic equation, defined only on the interface boundary, which describes the motion of the interface (see [24]). As previously in the level-set formulation, Dirichlet boundary conditions are used for $\mathbf{U}_{i}$ at the inlet and on the channel wall, while symmetry conditions are prescribed on the axis. At the outlet, the radial velocity is set to zero while the axial velocity satisfies a Neuman condition. In addition to these equations, continuity of velocity is imposed as well as the stress jump accounting for capillary forces at the interface

$$
\begin{gathered}
\llbracket \mathbf{U}_{i} \rrbracket_{r=H(z, t)}=0, \\
\llbracket \sigma_{i} \mathbf{n} \rrbracket_{r=H(z, t)}=\frac{\mathcal{C}}{\mathrm{Ca}} \mathbf{n},
\end{gathered}
$$

where the notation $\llbracket \cdots \rrbracket$ denotes the jump from the inner to the outer flow. The unit vector $\mathbf{n}$ normal to the interface pointing towards the outer fluid, the tangential vector $\mathbf{t}$ and the mean curvature $\mathcal{C}$ can be written as:

$$
\begin{aligned}
& \mathbf{n}=\frac{\left(1,-\partial_{z} H\right)}{\left(1+\left(\partial_{z} H\right)^{2}\right)^{1 / 2}}, \\
& \mathbf{t}=\frac{\left(\partial_{z} H, 1\right)}{\left(1+\left(\partial_{z} H\right)^{2}\right)^{1 / 2}}, \\
& \mathcal{C}=-\nabla \cdot \mathbf{n} .
\end{aligned}
$$

In order to carry out a global stability analysis we divide the flow variables $\mathbf{Q}=(\mathbf{U}, P, H)$ into the steady state $\overline{\mathbf{q}}_{i}=\left(\overline{\mathbf{u}}_{i}, \bar{p}_{i}, \bar{h}\right)$ and one small amplitude time-varying perturbation $\hat{\mathbf{q}}_{i}=\left(\hat{\mathbf{u}}_{i}, \hat{p}_{i}, \hat{h}\right)$ :

$$
\begin{aligned}
\mathbf{U}_{i}(r, z, t) & =\overline{\mathbf{u}}_{i}(r, z)+\varepsilon \hat{\mathbf{u}}_{i}(r, z) \exp (-\mathrm{i} \omega t) \\
P_{i}(r, z, t) & =\bar{p}_{i}(r, z)+\varepsilon \hat{p}_{i}(r, z) \exp (-\mathrm{i} \omega t) \\
H(z, t) & =\bar{h}(z)+\varepsilon \hat{h}(z) \exp (-\mathrm{i} \omega t)
\end{aligned}
$$

where $\omega \in \mathbb{C}$ is the complex mode frequency and $\varepsilon \ll 1$. The complex eigenfrequency is decomposed into its real (oscillating) and imaginary (growing or decaying) part $\omega=\omega_{r}+\mathrm{i} \omega_{i}$, such that a mode is unstable if $\omega_{i}>0$.

By injecting the flow decomposition into the governing equations and linearizing, we obtain a set of equations which describe the linear evolution of the perturbations. Note that the base flow and the linear stability computations are carried out through two different methods (see figure 2 for a sketch of the two different approaches), thus some preliminary steps need to be performed to do the stability analysis. 


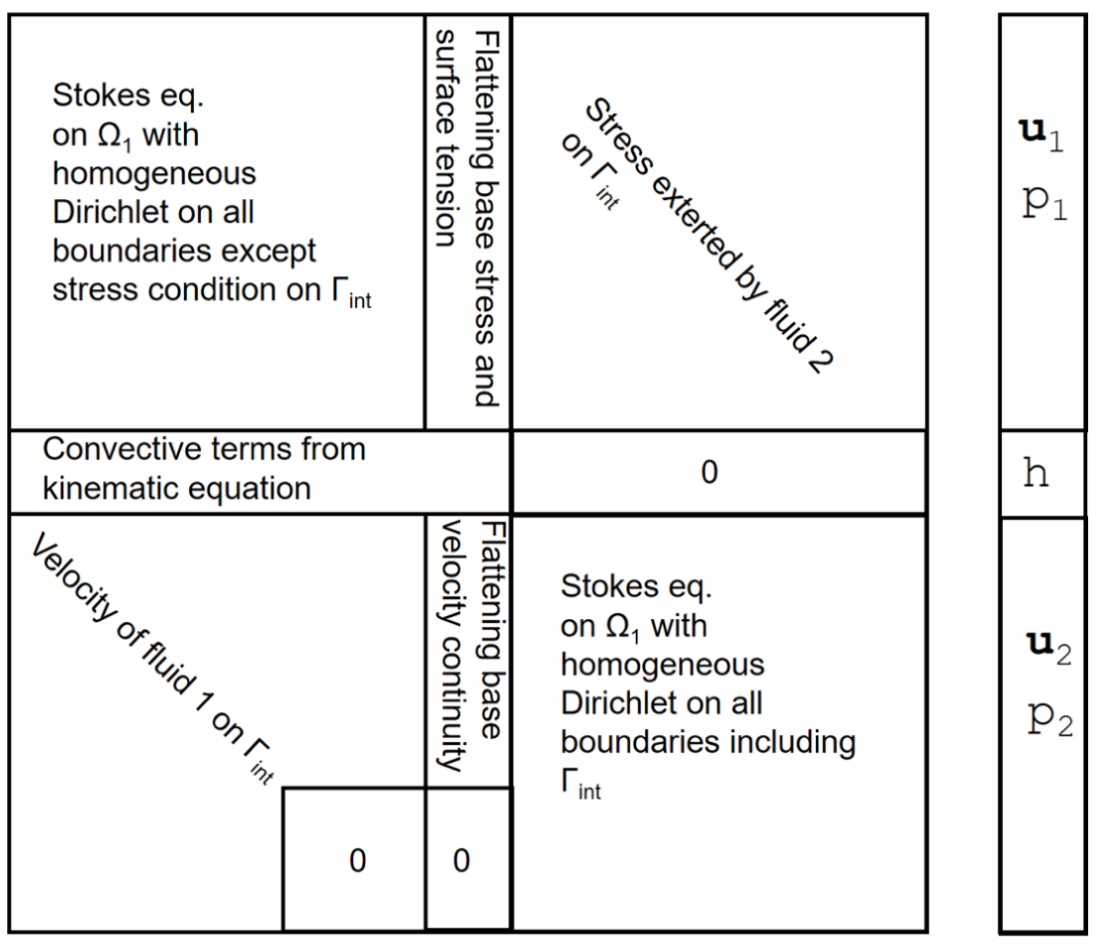

Figure 4. Structure of matrix A for the global stability analysis.

The computational domains are built using the level set interface position. Nonetheless, the obtained interface is characterized by small numerical oscillation caused by the interpolation of the isoline $\phi=0$. Therefore a fitting procedure was devised where an optimal fitting spline is computed by minimising an error indicator. Once the interface is discretized, the base flow can be easily computed in the two separated domains and the error due to the interface spline can be computed as $\int_{\Gamma_{\text {int }}} \overline{\mathbf{u}} \cdot \overline{\mathbf{n}} \mathrm{d} \Gamma / \int_{\Gamma_{\text {int }}} \mathrm{d} \Gamma$. The baseflow in the separated domains is computed by using a finite-element method, with cubic elements $P 3$ for the velocities and quadratic elements $P 2$ for the pressure, which is an higher order version of the standard Taylor-Hood elements (see [25] for details).

The governing equations for the linear evolution of the perturbations can be written as:

$$
\begin{aligned}
& 0=\nabla \cdot \hat{\sigma}_{\mathbf{i}} \\
& \nabla \cdot \hat{\mathbf{u}}_{i}=0
\end{aligned}
$$

$-\mathrm{i} \omega \hat{h}=-\bar{u}_{1} \frac{\partial \hat{h}}{\partial z}-\left(\hat{u}_{1}+\hat{h} \frac{\partial \bar{u}_{1}}{\partial r}\right) \frac{\partial \bar{h}}{\partial z}+\hat{v}_{1}+\hat{h} \frac{\partial \bar{v}_{1}}{\partial r}$

where $\hat{\sigma}_{i}=-\hat{p}_{i} \mathbb{I}+\mu_{i}\left(\nabla \hat{\mathbf{u}}_{i}+\left(\nabla \hat{\mathbf{u}}_{i}\right)^{T}\right)$ is the perturbed viscous stress tensor. Equation (26) is the linearized kinematic equation. Besides the no-slip conditions at the wall $\left(\left.\hat{u}_{2}\right|_{r=R}=\left.\hat{v}_{2}\right|_{r=R}=0\right)$ and the symmetry condition $\left(\left.\partial_{r} \hat{u}_{1}\right|_{r=0}=\left.\hat{v}_{1}\right|_{r=0}=0\right)$, the flow has to satisfy also the interface boundary conditions at the unperturbed interface $r=\bar{h}(z)$. We impose on the domain 2 the continuity of the velocity

$$
\hat{\mathbf{u}}_{1}+\hat{h} \partial_{r} \overline{\mathbf{u}}_{1}=\hat{\mathbf{u}}_{2}+\hat{h} \partial_{r} \overline{\mathbf{u}}_{2}
$$

while in domain 1 we impose the tangential and normal stress conditions

$$
\begin{gathered}
{\left[\left[\overline{\mathbf{t}}^{T} \bar{\sigma}_{i} \hat{\mathbf{n}}+\overline{\mathbf{t}}^{T} \hat{\sigma}_{i} \overline{\mathbf{n}}+\hat{\mathbf{t}}^{T} \bar{\sigma}_{i} \hat{\mathbf{n}}+\hat{h}^{T} \frac{\partial \overline{\bar{n}}_{i}}{\partial r} \overline{\mathbf{n}}\right]\right]_{r=\bar{h}(z)}=0} \\
{\left[\left[\overline{\mathbf{n}}^{T} \hat{\sigma}_{i} \overline{\mathbf{n}}+2 \hat{\mathbf{n}}^{T} \bar{\sigma}_{i} \overline{\mathbf{n}}+\hat{h} \overline{\mathbf{n}}^{T} \frac{\partial \bar{\sigma}_{i}}{\partial r} \overline{\mathbf{n}}\right]\right]_{r=\bar{h}(z)}=\mathrm{Ca}^{-1}\left(\overline{\mathbf{n}}^{T} \hat{\mathcal{C}} \overline{\mathbf{n}}+2 \hat{\mathbf{n}}^{T} \overline{\mathcal{C}} \overline{\mathbf{n}}\right)}
\end{gathered}
$$

where we recall that the notation $\llbracket \cdots \rrbracket$ denotes the jump from the inner to the outer flow. The geometric properties of the perturbed interface are obtained by introducing the flow decomposition (23) into the relations (20)-(22) and linearising. In particular, the perturbed normal vector $\hat{\mathbf{n}}$ and curvature perturbation $\hat{\mathcal{C}}$ are written as:

$$
\begin{gathered}
\hat{\mathbf{n}}=-\frac{\partial_{z} \hat{h}\left(\partial_{z} \bar{h}, 1\right)}{\left(1+\left(\partial_{z} \bar{h}\right)^{2}\right)^{3 / 2}}, \\
\hat{\mathcal{C}}=-\frac{\hat{h}}{\bar{h}^{2}\left(\left(\partial_{z} \bar{h}\right)^{2}+1\right)^{1 / 2}}-\frac{1}{\left(\left(\partial_{z} \bar{h}\right)^{2}+1\right)^{3 / 2}} \partial_{z z} \hat{h} \\
+\left(\frac{3 \partial_{z z} \bar{h} \partial_{z} \bar{h}}{\left(\left(\partial_{z} \bar{h}\right)^{2}+1\right)^{5 / 2}}-\frac{\partial_{z} \bar{h}}{\bar{h}\left(\left(\partial_{z} \bar{h}\right)^{2}+1\right)^{3 / 2}}\right) \partial_{z} \hat{h} .
\end{gathered}
$$

At the inlet boundary we impose an homogeneous Dirichlet conditions on the radial velocity $\hat{v}$ and interface displacement $\hat{h}$, while we impose a Neumann condition on the streamwise velocity $\hat{u}$. Finally at the outlet we add a sponge region where the velocities and the height function are forced smoothly towards zero (see details in appendix A). 
Equations (24)-(26), together with the boundary conditions are discretized in space similarly to the base-flow ( $P 3-P 2$ elements). The interface perturbation $\hat{h}$ has been discretized with a $P 2$ element defined on the interface boundary. The meshes as well as the discrete matrices resulting from Galerkin finiteelement method are generated with the software FreeFem ++ [26], leading to a generalized eigenvalue problem

$$
\mathbf{A x}=-\mathrm{i} \omega \mathbf{B} \mathbf{x},
$$

where $\mathbf{x}$ is a vector containing all the discrete unknowns of the problem, i.e. velocities and pressure for the two fluids and the interface perturbation $\hat{h}$. The matrix A contains the discretization of the differential operators and includes the boundary conditions. To build this matrix, the following steps have to be performed: (i) we build the matrices $\mathbf{A}_{i}$ for the separated domains, (ii) the kinematic equation is discretized, (iii) we build the block off-diagonal matrices and (iv) finally we assemble the four matrices into the global one. A sketch of the matrices' structure of the matrices is shown in figure 4. Matrix $\mathbf{A}_{1}$ contains the discretization of the Stokes equations for the inner fluid with a stress condition on the interface, while matrix $\mathbf{A}_{2}$ contains the discretization of the Stokes equations for the outer fluid, where we have a Dirichlet boundary condition for the interface velocity. The two sub-problems are coupled together by imposing at the discrete level the stress exerted by fluid 2 on fluid 1 with an upper-diagonal matrix and the continuity of velocity at the interface as a lower-diagonal matrix. The matrix $\mathbf{B}$ is in principle equal to a mass matrix on the degree of freedom related to the interface position and null anywhere else. Nonetheless, for numerical stability reasons, at the left-hand side of the perturbed Stokes equation (24), a small term $-\mathrm{i} \epsilon \omega \hat{\mathbf{u}}_{i}$ is added. The value $\epsilon=10^{-5}$ has been chosen in such way that the first four digits of the most unstable eigenvalue did not change with the addition of this term. The eigenvalue problem (32) is solved with a KrylovShur method along with a shift-invert strategy by using the numerical library SLEPc ([27]).

\section{Validation: stability of a parallel flow}

The global stability framework is now validated for a parallel flow with a fully-developed velocity profile, taking as computational domain of finite length $L$. Therefore the interface height is constant $\left(\bar{h}(z)=H_{\text {in }}=H_{\text {out }}\right)$, the base flow velocity field depends only on the $r$-coordinate $\overline{\mathbf{u}}=(\bar{u}(r), 0)$ and the pressure field $\bar{p}$ respects both the pressure jump at the interface $\bar{p}_{1}-\bar{p}_{2}=\mathrm{Ca}^{-1} \overline{\mathcal{C}}=\mathrm{Ca}^{-1} H_{\text {out }}^{-1}$ and the pressure gradient $\partial_{z} \bar{p}=-4\left(1-H_{\text {out }}^{2}\right)$. The parallel flow case allows us to validate the global stability tools against the results of a local stability analysis. As reference we consider the lubricated local analysis performed by [7] and the exact local analysis performed by [10] for the creeping flow limit. Note that in [10], the flattening contribution coming from the disturbed interface position in velocity and stress continuity was forgotten. We have therefore implemented a Chebyshev discretization of

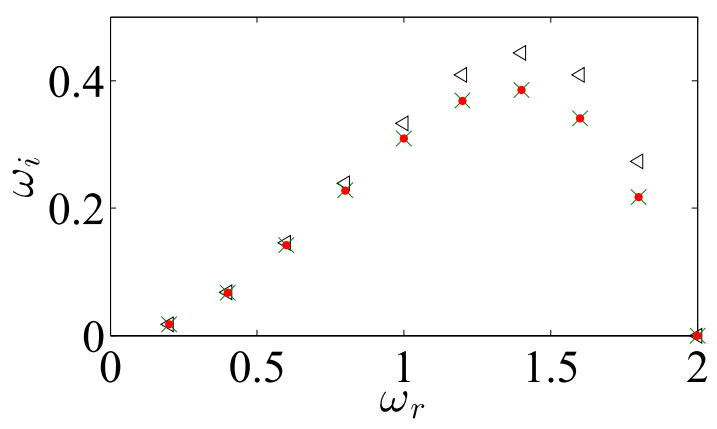

(a)

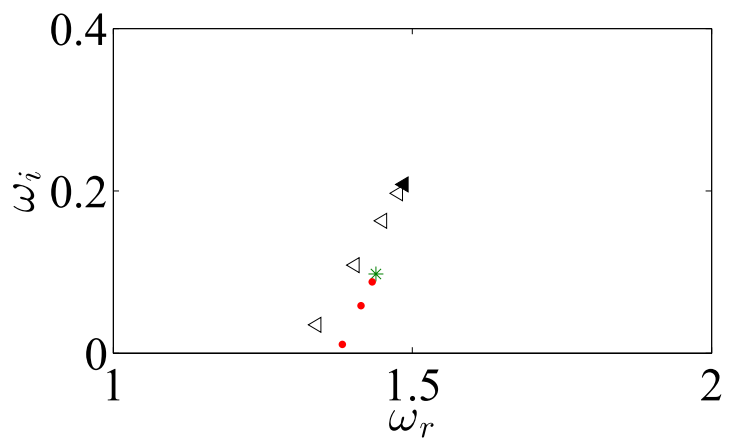

(b)

Figure 5. Comparison of the global and local stability results for a parallel flow case with $H_{\text {out }}=0.5, \lambda=1, \mathrm{Ca}=1 / 20$ and $L=10 \pi$. At inlet and outlet we impose different boundary conditions: (a) periodic (b) Dirichlet $\hat{v}=0$ and $\hat{h}=0$. The white triangles $(\triangleleft)$ are the solution of the global lubrication stability analysis inspired by the dispersion relation in [7] (see appendix B), respectively with periodic boundary conditions (a), and Dirichlet conditions (b). The green crosses $(X)$ are the exact solutions of the local analysis performed by [10] (with additional flattening terms missing in his derivation) computed from our Chebyshev code. The red dots ( $\bullet$ ) are the solution of the global stability analysis, while the filled black triangle ( 4 in figure (b) is the saddle point of the dispersion relation in [7] and the star (*) in (b) the saddle point of the corrected dispersion relation in [10] including the missing flattening terms. (a) Periodic boundary conditions. (b) Dirichlet boundary conditions.

these linearized $1 \mathrm{D}$ equations as a correction to the dispersion relation in [10]. This numerical code also easily allowed us to determine the saddle point of dispersion relation. This local stability code is validated comparing the green crosses and the red points in figure 5(a), where the imaginary (growing or decaying) part $\omega_{i}$ of the obtained eigenvalues is plotted as a function of its real (oscillating) part $\omega_{r}$, such that a mode is unstable if $\omega_{i}>0$.

Figure 5 shows the stability for a parallel flow case with interface height $H_{\text {out }}=0.5$, viscosity ratio $\lambda=1$, Capillary number $\mathrm{Ca}=1 / 20$ and $L=10 \pi$. As a first validation we impose periodic boundary conditions at the right and left boundaries. As expected the global analysis spectrum recovers the local analysis dispersion relation. In figure 5(a) we see that the global analysis (red dots) is in excellent agreement with the corrected analysis in [10] (green crosses) while the lubrication hypothesis in [7] (black triangles) yields a slight overestimation of the growth rate. 

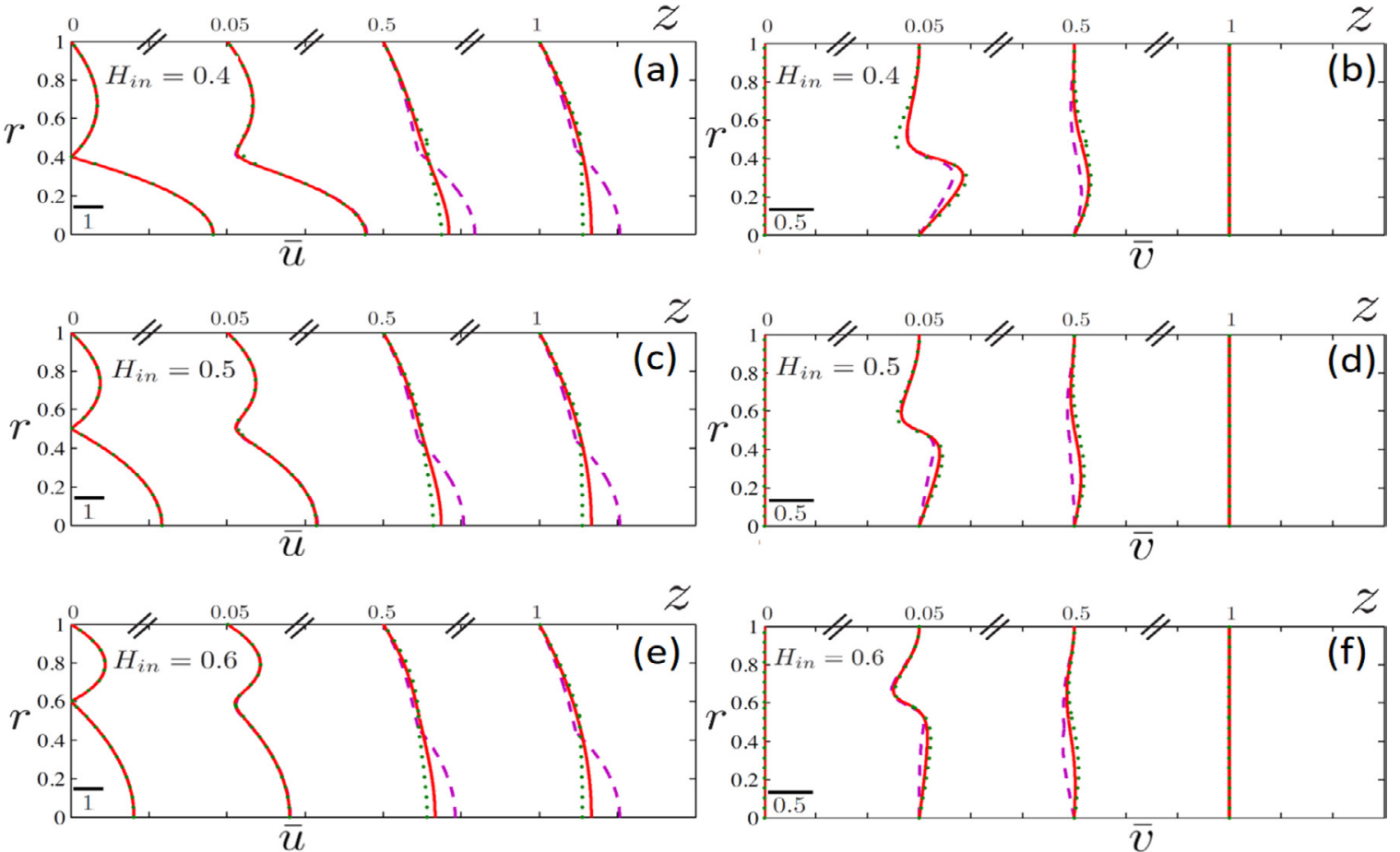

Figure 6. Axial $\bar{u}((\mathrm{a}),(\mathrm{c})$ and (e)) and radial $\bar{v}((\mathrm{~b})$, (d) and (f)) velocity profiles for different sections along $z=\{0,0.05,0.5,1\}$. The parameters $Q_{2}=1.18$ and $\mathrm{Ca}=1$ are kept constant, while $H_{\text {in }}$ is varied from top to bottom from 0.4 ((a) and (b)) via 0.5 ((c) and (d)) to 0.6 ((e) and (f)). Colors represent different $\lambda-Q$ combinations : $\lambda=1 / 5 ; Q_{1}=1.44$ (purple dashed line), $\lambda=1 ; Q_{1}=0.916$ (red line) and $\lambda=5 ; Q_{1}=0.812$ (green dotted line)

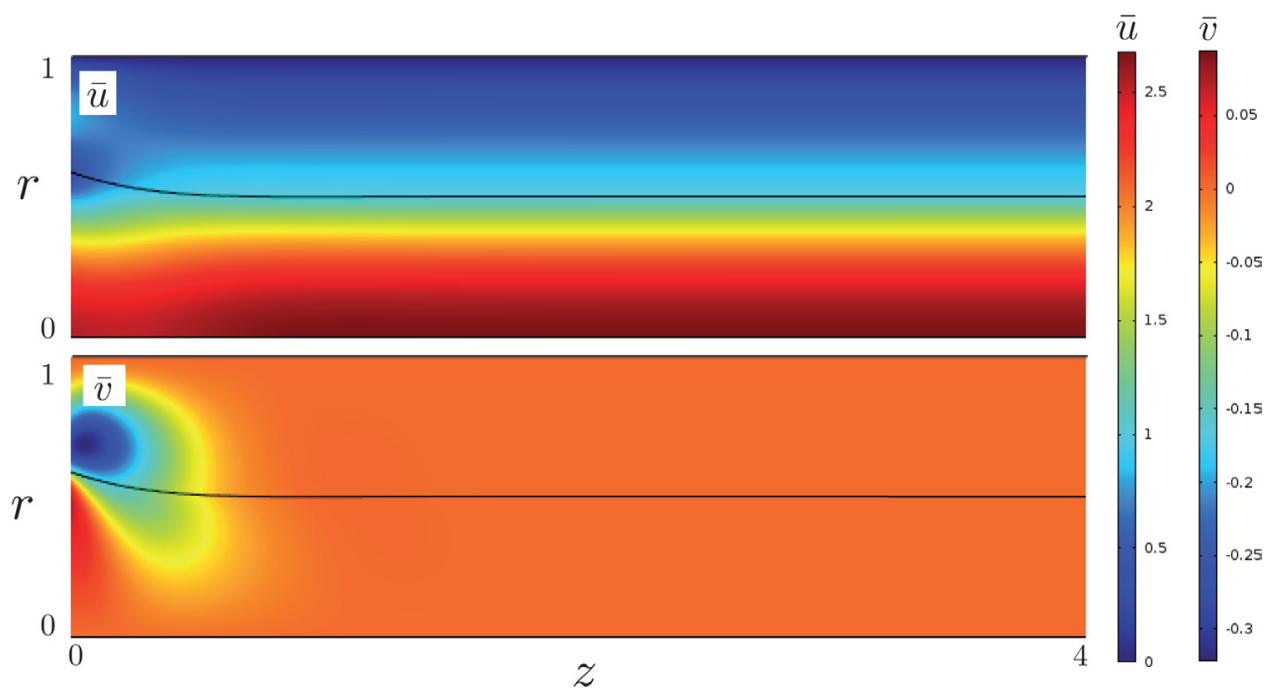

Figure 7. Streamwise velocity $\bar{u}$ (top) and radial velocity $\bar{v}$ for a co-axial flow with $Q_{1}=1.44, Q_{2}=1.18, H_{\text {in }}=0.6, \lambda=1 / 5$ and Ca $=1$.

We then consider a finite computational domain, where we impose the Dirichlet boundary conditions $\hat{v}=\hat{h}=0$ both at the inlet and the outlet. The global modes for an $z$-independent system, such as the one studied in this section, are known to be reminiscent of the absolute frequency of the system. In the limit of an infinite streamwise extension of the domain $(L \rightarrow \infty)$, a continuous branch of the spectrum emanates from each saddle point $\omega_{0}$, as explained in $[28,29]$. In contrast, when we consider a computational box of finite size a discrete spectrum of global modes which satisfy the boundary conditions is expected. In figure 5(b) we see that the absolute mode does indeed mark a point from which the sequence of global modes emerges. The global modes behaviour is also confirmed in the lubrication hypothesis case (black triangles), where we perform a global analysis by using a simplified model derived by taking advantage of the polynomial dispersion relation (see appendix B for details) pertaining when this approximation is enforced. Figure 5 shows that in the lubrication hypothesis, both the frequency and growth rate of the global modes are overestimated, for both sets of streamwise boundary conditions. 


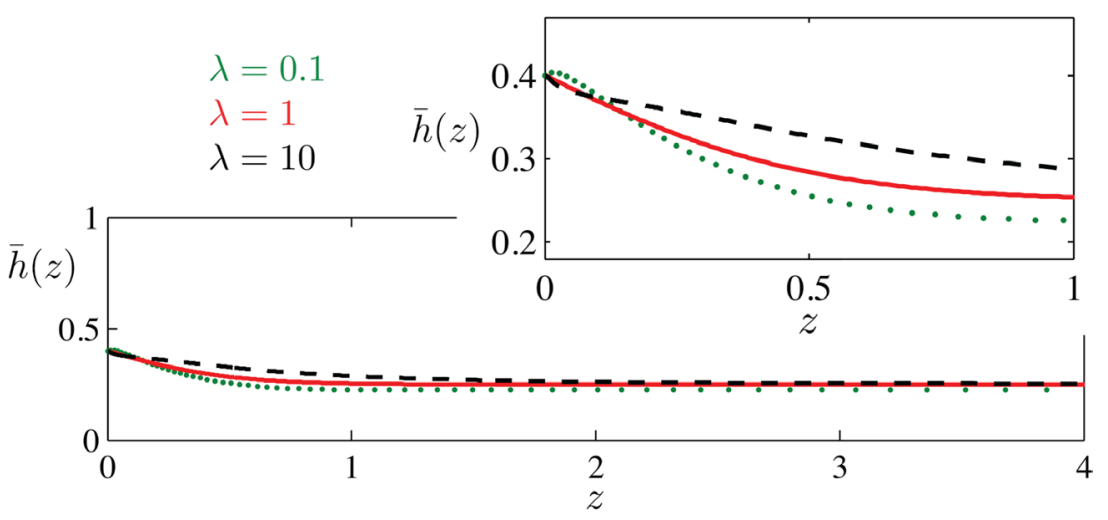

Figure 8. Influence of the viscosity ratio on the interface displacement. We impose at the inlet $Q_{1}=0.2029, Q_{2}=1.4726$ and $H_{\text {in }}=0.4$ and we determine the interface height for different viscosity ratio $\lambda=\{0.1,1,10\}$. When the outer flow is more viscous $\lambda<0.1$ (green dotted line) we can observe the interface height $h(x)$ is not monotonic, and it reaches the fully developed state faster than when the inner flow is more viscous $\lambda=10$ (black dashed line).
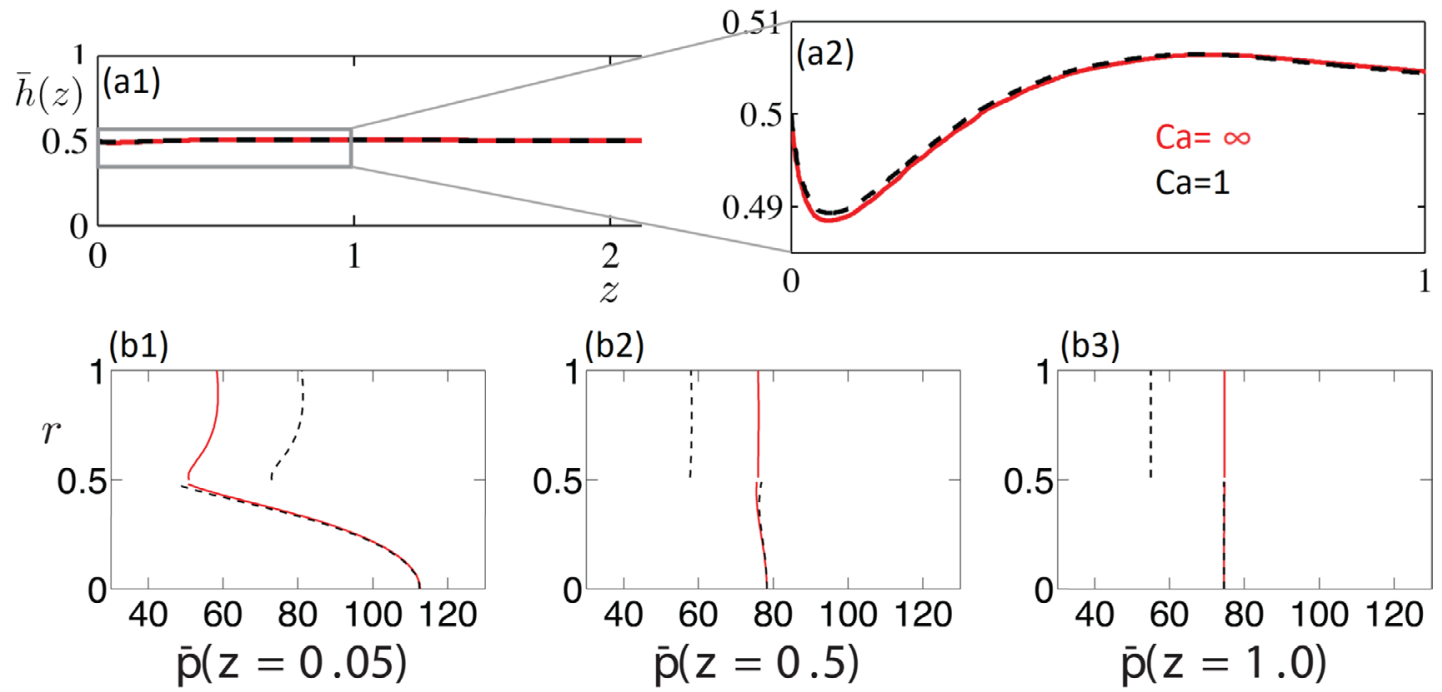

Figure 9. Influence of the surface tension on the base flow; $H_{\text {in }}=0.5, H_{\text {out }}=0.5 \lambda=5.0, Q_{1}=0.812$ and $Q_{2}=1.18$. The red lines correspond to the case without surface tension $\mathrm{Ca}=\infty$, while the black dashed lines were obtained with $\mathrm{Ca}=1$. In (a), the interface is represented with a zoom on the entry region. In (b), the pressure profiles are given at different streamwise stations: $z=0.05$ (b1), $z=0.5$ (b2) and $z=1(\mathrm{~b} 3)$.

\section{Base flow of co-axial streams}

Given the very large parameter space describing the possible base flows, we concentrate at present on a few sets of interesting parameters. The base flow is obtained as described in section 2.2. We use a rectangular computation domain with radius 1 and length $L=10$. The domain is long enough to allow the flow to reach the fully developed state. The inlet profile, defined in (7)-(8) assumed parabolic in the inner layer and parabolic-logarithmic in the outer layer, introduces a region of local deficit which gradually recedes towards a double-parabolic channel flow profile developed downstream.

The base flow changes rapidly as it evolves downstream. In figure 6 we plot the streamwise and radial velocity for different streamwise stations. In the region close to the nozzle we notice that a region with negative radial velocity appears,

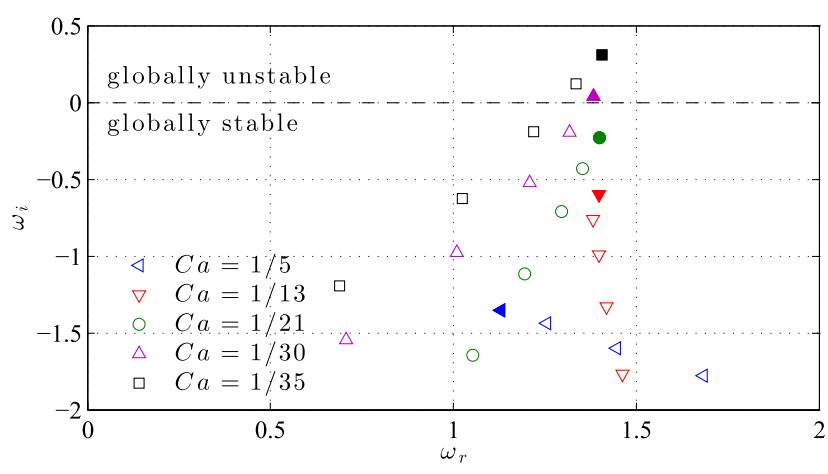

Figure 10. Eigenvalues for $H_{\text {in }}=0.5, H_{\text {out }}=0.4, \lambda=5$ and decreasing value of $\mathrm{Ca}$. Each combination of color and marker is associated with a different value of $\mathrm{Ca}$. The filled markers are the most unstable eigenvalue for that capillary number. 


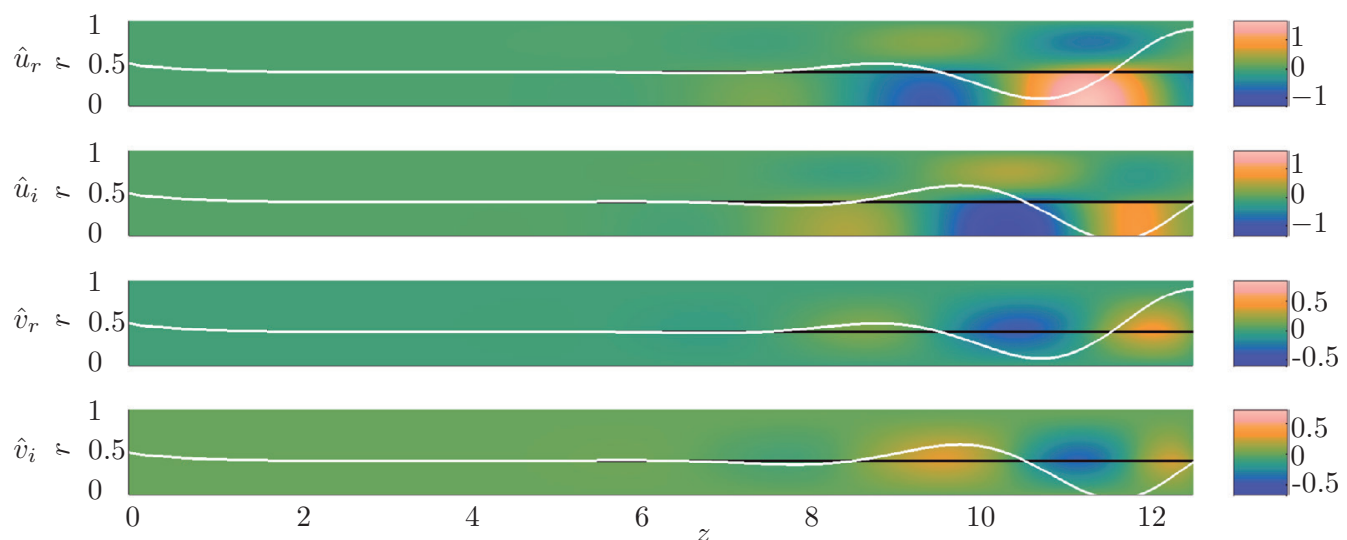

Figure 11. Real and imaginary part of the eigenfunctions of unstable mode for $H_{\text {in }}=0.5, H_{\text {out }}=0.4, \lambda=5$ and Ca $=1 / 30$. Comparison unperturbed interface (black line) and perturbed interface (white line). The color map is scaled with the maximum real amplitude of $h$ of the eigenfunction.

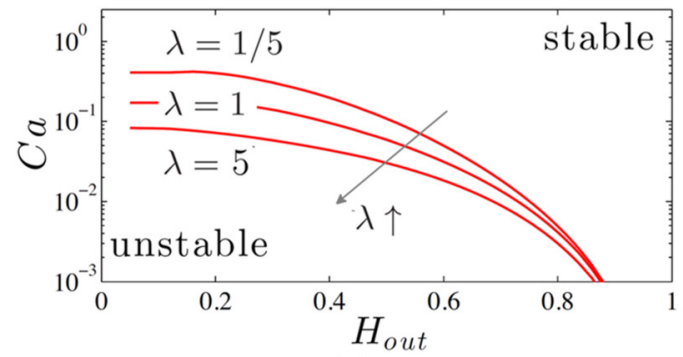

(a) $\lambda \uparrow$

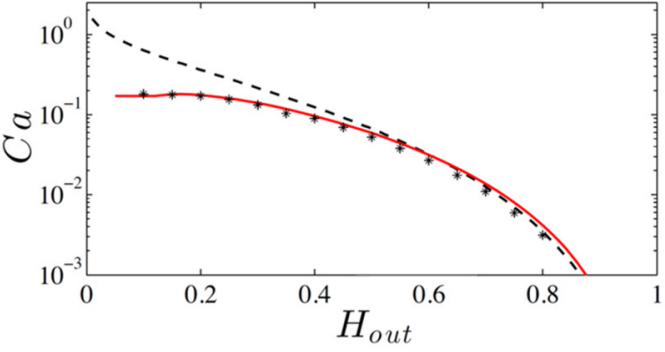

(c) $\lambda=1$

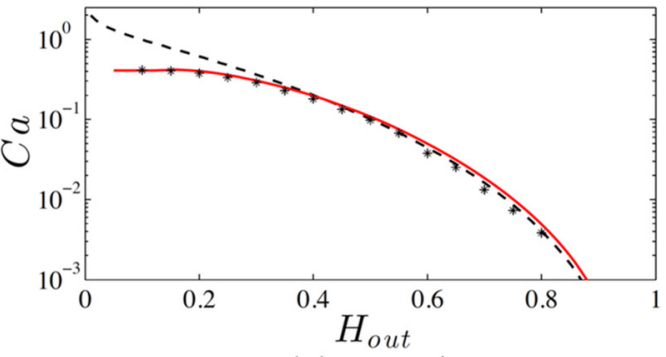

(b) $\lambda=1 / 5$

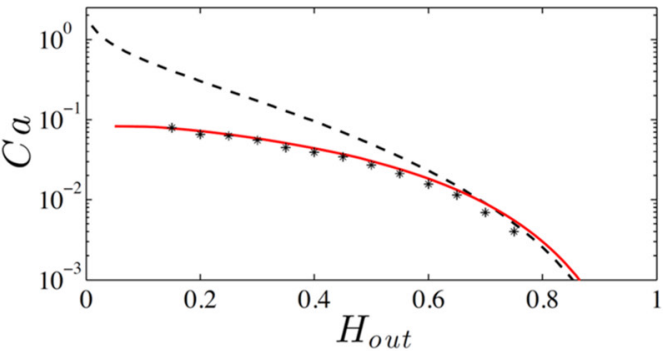

(d) $\lambda=5$

Figure 12. (a) Absolute/convective transition curves from the modified dispersion relation of [10] for $\lambda=\{1 / 5,1,5\}$. (b)-(d) phase diagram of the instability in the $\left(H_{\text {out }}, \mathrm{Ca}\right)$ plane for a fixed value of $H_{\text {in }}=0.5$ and different value of viscosity ratio $\lambda=\{1 / 5,1,5\}$. The black dashed lines represent the local lubrication analysis [7], the red lines are the absolute/convective transition curves from the dispersion relation of [10] with the addition of the flattening terms. The black points are the results of our global analysis. Above the curves, the system is stable, while below it is unstable.

which quickly diminishes, as confirmed by the velocity maps of figure 7. The fully-developed piecewise Poiseuille flow develops in about a radius. The necessary space to obtain a completely developed flow does not seem to depend on the initial confinement $H_{\text {in }}$ of the co-axial flow.

The base flow and the interface location depend on the Capillary number $\mathrm{Ca}$, the viscosity ratio $\lambda=\mu_{1} / \mu_{2}$, flow rate ratio $Q=Q_{1} / Q_{2}$ and aspect ratio $H_{\text {in }}$. Figure 8 underlines how the streamwise velocity in the entry region and the height of the interface depends on the viscosity ratio. Changing the viscosity ratio, the monotonicity of the interface position changes. If the outer flow is more viscous $(\lambda<1)$ the interface reaches the fully developed state always in a nonmonotonous way. Moreover, for the same imposed inlet flow rate $Q_{1}$ and $Q_{2}$, if $\lambda<1$ the flow evolves faster, reaching the

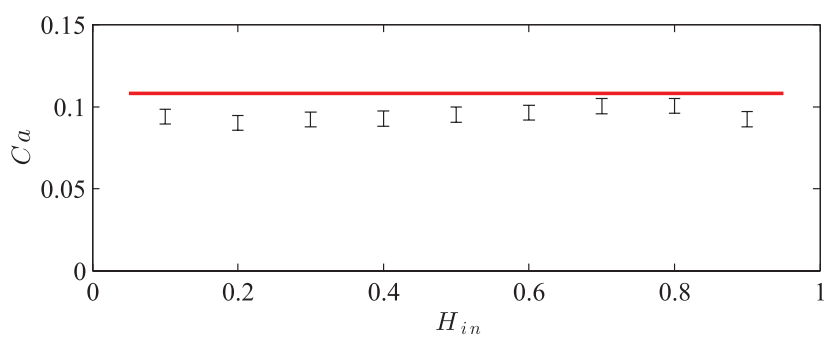

Figure 13. Neutral capillary number as a function of the nozzle diameter $H_{\text {in }}$ for a fixed value of the degree of confinement of the fully developed flow $H_{\text {out }}=0.5$ and viscosity ratio $\lambda=1 / 5$. The red line is the theoretical line separating the dripping region (above) and the jetting region (below) predicted by the local analysis ([10]). The black points are the global modes with their respective error bars obtained by changing the interpolation procedure of section 2.3 . 

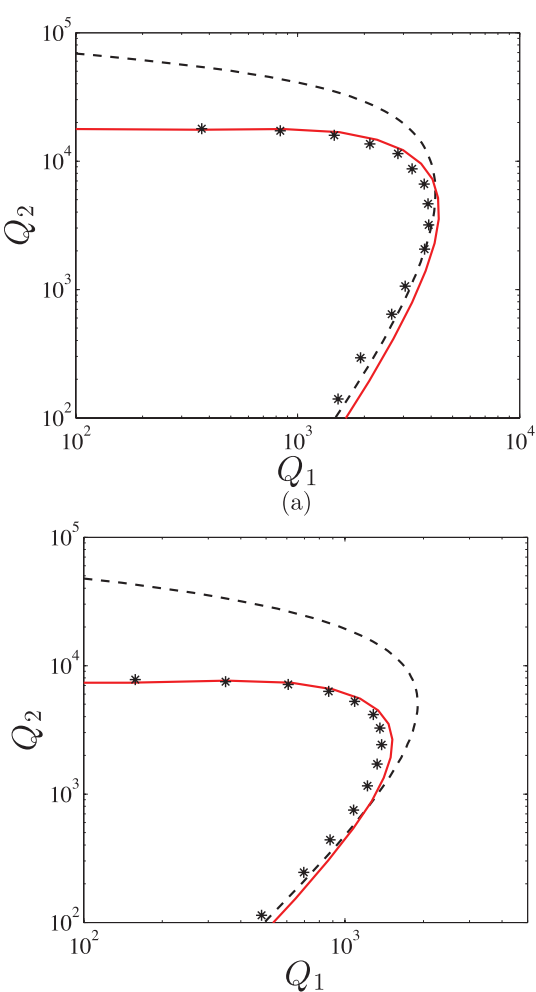

(c)

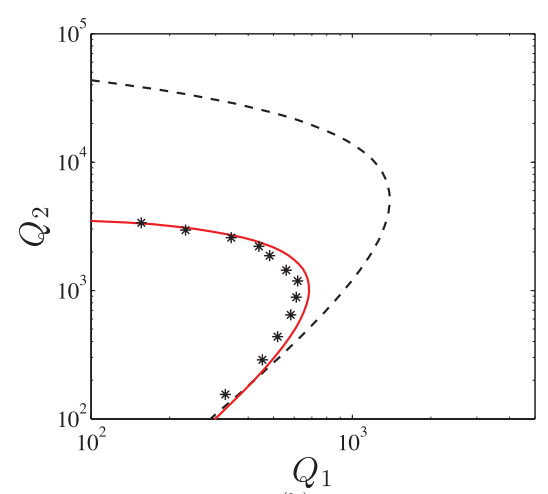

(b)

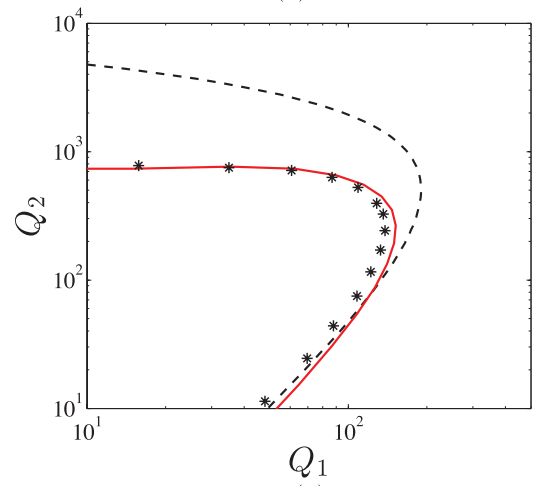

(d)

Figure 14. Dynamic behavior in $\left(\tilde{Q}_{1}, \tilde{Q}_{2}\right)$ plane and stable/unstable transition. The black dashed lines correspond to the lubrication coaxial analysis performed by Guillot et al [7]. The red line represent the no-inertial analytic solution found by Herrada et al [10] with the addition of the flattening terms in the analysis. The black points are the results of our global stability analysis. The flow rates are expressed in $\left(\mu \mathrm{l} \mathrm{h}^{-1}\right)$, the outer radius is $R=275 \mu \mathrm{m}$ while the outer viscosity $\mu_{2}=0.235 \mathrm{~Pa}$. The nozzle radius is $H_{\text {in }}=0.5$ for all transition illustrated.

fully developed state earlier than when the inner flow is more viscous.

Surprisingly, the surface tension plays a minor role on the base flow. Increasing the surface tension, for the same operating parameters, the interface position does not change. As consequence also, the velocity field is not effected by an increase of surface tension. Minor differences can be noticed only when the inner flow is more viscous than the outer (see figure 9). Nonetheless, the pressure jump, according to the Laplace law, increases if the surface tension increases.

\section{Stability of the spatially developing flow}

We now investigate the stability of the steady spatially developing flow described in the previous section.

Figure 10 shows how the surface tension affects the stability of the flow. We consider a co-axial flow with $H_{\text {in }}=0.5$, $H_{\text {out }}=0.4, \lambda=5$ and we decrease the Capillary number. In the $\left(\omega_{r}, \omega_{i}\right)$-plane we plot the eigenvalues obtained by the global stability described in section 2.3. The frequency and growth-rate of the eigenvalues are given by the real and complex part of $\omega=\omega_{r}+\mathrm{i} \omega_{i}$ respectively. We observe a bifurcation from stable $\left(\omega_{i}<0\right)$ to unstable $\left(\omega_{i}>0\right)$ between $\mathrm{Ca}=1 / 21$ and $\mathrm{Ca}=1 / 30$. The growth rate increases if the Capillary number decreases. If $\mathrm{Ca}<1 / 35$ there are several unstable modes, in analogy with the general picture shown in section 3. The spatial shape of the unstable mode can be seen in figure 11. The streamwise and radial components of the oscillation $\hat{u}, \hat{v}$ and the perturbed interface are amplified downstream.

As revealed in figure 12, we now track the neutral curve in the $\left(H_{\text {out }}, \mathrm{Ca}\right)$-plane, where the transition separating the unstable region (below the points) and the stable region (above the points) are reported, for a fixed inlet nozzle position $H_{\text {in }}=0.5$ and varying viscosity ratios $\lambda=\{1 / 5,1,5\}$. In the $\left(H_{\text {out }}, \mathrm{Ca}\right)$-plane we can also plot the absolute/convective unstable transition obtained from the local stability analysis of the fully developed flow for the same operating parameters. Convective instabilities, which are convected downstream while growing in amplitude, are in this setting expected to be associated to globally stable flows, while absolute instabilities, which grow and travel backwards are likely to correspond to globally unstable flows. More specifically, we report in figures 12(b)-(d) the A/C transition curves found by [7] (black dashed lines) and the modified dispersion relation in [10] (red lines). Observe that, in all cases that we have analyzed, the global stability predicts a more stable flow than the lubrication and Stokes equations-based local analyses. In particular we notice that for small value of the degree of confinement $H_{\text {out }}$ the Stokes parallel flow analysis and the global stability have the same qualitative and qualitative behaviour, while the lubrication local stability fails in the prediction of the convective/ absolute transition. In contrast the lubrication and exact local 
solutions slightly overestimate the global results when the confinement ratio is large $\left(H_{\text {out }} \sim 0.7\right)$ and the flow becomes more sensitive to the presence of the wall. The viscosity ratio has a large impact on the stability properties of the flow. In particular, the flow is more and more stable as the viscosity ratio $\lambda$ decreases. For reference, the absolute/convective transition curves from the modified dispersion relation in [10] have been reported in figure 12 (a) for $\lambda=\{1 / 5,1,5\}$.

We now let the nozzle radius $H_{\text {in }}$ vary. From the different base flow behaviour depicted in section 4 , a significant effect on the global stability could be expected. Surprisingly, for a fixed $H_{\text {out }}$ the critical capillary number has been found almost insensitive to the nozzle dimension, as shown in figure 13. The error bars in the figure are computed by keeping fixed the mesh resolution and by just changing the interface interpolation spline. We estimate an average error of about $10 \%$ due to the interface interpolation procedure. We can see that the fitting error is comparable to the influence of the nozzle position. Based on these considerations, we kept fixed $H_{\text {in }}=0.5$ in the subsequent part of the work.

Following the guidelines in [10] and [7], we represent the stability results also in the $\left(\tilde{Q}_{1}, \tilde{Q}_{2}\right)$ operational plane, for values of the parameters common in microfluidic devices (see figure 14). This representation is particularly useful for comparison with experimental data, since in experiments the flow is modified by changing the flow rates. At fixed $\tilde{Q}_{2}$, increasing $\tilde{Q}_{1}$ increases the jet velocity allowing the instability to have more convection, and this behaviour promotes continuous jets. As observed above, for small value of the outer flow rate $\tilde{Q}_{2}$, that correspond to large value of $H_{\text {out }}$, the local stability analysis slightly overestimates the global results. We can conclude that the dripping to jetting transition is a process mostly dominated by the intrinsic properties of the fully developed streams, and that the development of the flow in the entry region has a small stabilizing effect on the flow. In particular, the role of the entry region seems to be more relevant for low values of the external flow rate. It is noteworthy that also available experimental data (see $[10,11])$ shows that in this regime the flow is more stable compared to the local analysis predictions.

\section{Conclusion}

In this study, we have analysed the influence of the entry region on the dripping to jetting transition in a coflowing streams device.

We have first investigated the effect of several parameters such as the flow rate ratio $Q$, the viscosity ratio $\lambda$ and the capillary number $\mathrm{Ca}$ on the flow behaviour of the steady continuous jets solution. In addition, we characterized the effect of geometry by varying the nozzle radius $H_{\text {in }}$, showing that the flow varies rapidly (in about one radius) from the nozzle velocity profile to the parallel fully developed flow.

In the second part, we have performed a global stability analysis of the spatially evolving two phase flow. For high external flow rate value, the global stability analysis almost recovers the same results of the exact local analysis. This implies that for low degrees of confinement, the dripping to jetting transition is a process dominated by the intrinsic properties of the developed streams and not by the complex flow evolution in the entry region. In contrast, for small external flow rate, or high degree of confinement, the flow is more sensitive to the presence of the wall and becomes slightly more stable than predicted by the local theory. We also showed that the dripping to jetting transition is not affected by the geometrical details such as the nozzle dimension.

Despite an improved accuracy ensured by the global character of our analysis with respect to a local spatio-temporal analysis of the fully developed profile, the main limitation of the present study remains its linearity. Whether the nonlinear development of the instability and the subsequent pinch-off in the near-nozzle region have an importance on the frequency of droplet production remains unaddressed, calling for future non-linear analyses. Whether such a nonlinear analysis can also account for the failure of the local absolute/convective frequency prediction [30] in the dripping regime, remains an open issue.

Another natural perspective consists in taking account inertia both in the base flow computation as well as in the global stability analysis. Inertia is likely to elongate the entry length, thereby possibly reinforcing the influence of the inlet condition and nozzle geometry. Finally, the influence of surface rheology and soluto-capillary effects on the base flow development and its instability deserves also to be considered.

\section{Acknowledgments}

Support for this work by the ERC grant (ERC-2011-StG, PE8 SIMCOMICS 280117) and the FNS (Fond National Suisse) grants 200021-132323 and 200020-152957 are gratefully acknowledged.

\section{Appendix A. Sponge region at the outlet of the perturbed computational domain}

The sponge region extends from $L_{\mathrm{bf}}=L+2.5$ to $L_{\mathrm{gs}}=L+5$ and in this region we impose a forcing both in the momentum and kinematic equation:

$$
\xi_{f}=\frac{\alpha f}{1+\exp \left(\frac{1}{z^{\prime}-1}+\frac{1}{z^{\prime}}\right)}
$$

where $z^{\prime}=\frac{z-L_{\mathrm{bf}}}{L_{\mathrm{gs}}-L_{\mathrm{bf}}}, \alpha=50$ is the force strength parameter and $f$ indicates respectively $\{\mathbf{u}, h\}$.

\section{Appendix B. Toy model for the global stability analysis of coaxial jets under the lubrication hypothesis}

Under the lubrication hypothesis, the dispersion relation for the coaxial jets has a polynomial form [7]:

$$
\omega(k)=\alpha k+\mathrm{i} \beta\left(k^{2}-k^{4} h^{2}\right)
$$




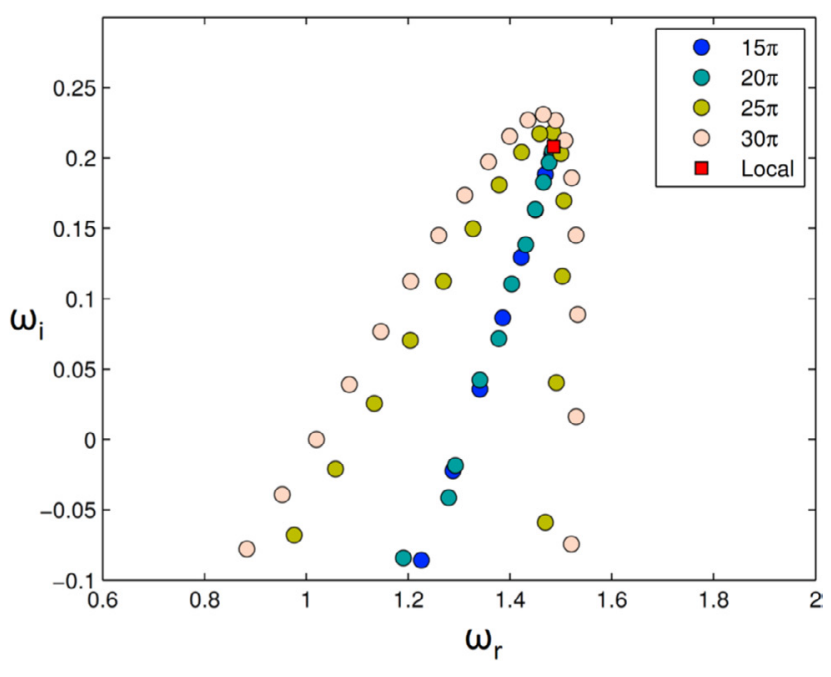

Figure B1. Influence of the length of the domain $L$ on the eigenvalues with $\alpha=1$ and $\beta=0.4438$, i.e. degree of confinement $h=0.5$, viscosity ratio $\lambda=1$ and Capillary number $\mathrm{Ca}=1 / 20$.

where $\alpha$ and $\beta$ are functions of the flow parameters $(\mathrm{Ca}, \lambda$ and $Q)$ and $h$ is the interface position.

In such cases, it is possible to recover the underlying PDE from the dispersion relation. Let us consider a general plane wave solution $\phi(z, t)=\phi_{0} \exp (\mathrm{i} k z-\mathrm{i} \omega t)$, (note that $\phi$ does not refer here to the level set function, but rather to an arbitrary function) where the differential operators become:

$$
\begin{gathered}
\frac{\partial}{\partial t}=-\mathrm{i} \omega \\
\frac{\partial^{n}}{\partial z^{n}}=(\mathrm{i} k)^{n} .
\end{gathered}
$$

By manipulating equations (B.1), (B.2) and (B.3) we obtain:

$$
\frac{\partial \phi}{\partial t}=-\beta\left(\frac{\partial^{2} \phi}{\partial z^{2}}+h^{2} \frac{\partial^{4} \phi}{\partial z^{4}}\right)-\alpha \frac{\partial \phi}{\partial z} .
$$

This 1D problem can be used to carry out a global stability analysis and to investigate the effect of the streamwise confinement. In the spirit of a global analysis, let us consider a solution in the following form $\phi(z, t)=\phi \hat{(z)} \exp (-i \omega t)$, where only the time dependence is sought in normal mode form. We obtain the following eigenvalue problem:

$$
-\mathrm{i} \omega B \hat{\phi}=A(\alpha, \beta) \hat{\phi}
$$

where $A=-\beta\left(\frac{\partial^{2}}{\partial z^{2}}+h^{2} \frac{\partial^{4}}{\partial z^{4}}\right)-\alpha \frac{\partial}{\partial z}$.

Problem (B.5) is numerically solved with a Chebyshev spectral collocation method in a physical domain ranging from $0 \leqslant z \leqslant L$, with inlet and outlet homogeneous Dirichlet boundary conditions $\phi(0)=\phi(L)=0$.

As already noted by [29], there is an upper limitation on the lenght $L$ of the computational box. In figure B1 we can see that when $L$ is larger than $20 \pi$ the global spectrum is not aligned on a single branch, but instead the eigenvalues arrange themselves on a bell shaped curve which passes above the location of the true global modes. Heaton et al [29] suggest that this phemonenon is caused by a combination of the non-normality of the linear operator and issues of numerical precision. Since the global mode is expected to grows exponentially in the axial direction, if the computational box is too large the global mode can not be resolved accurately. Heaton et al [29] proposed that $\left|k_{0 i}\right| L<c$ where $c$ is a constant that depends on the considered case.

\section{ORCID iDs}

L Augello (1) https://orcid.org/0000-0001-8434-7260

A Fani (ㄱ https://orcid.org/0000-0002-7082-8087

F Gallaire (1) https://orcid.org/0000-0002-3029-1457

\section{References}

[1] Stone H A 1994 Ann. Rev. Fluid Mech. 26 65-102

[2] Eggers J and Villermaux E 2008 Rep. Prog. Phys. 71036601

[3] Baroud C N, Gallaire F and Dangla R 2010 Lab Chip 10 2032-45

[4] Cramer C, Fischer P and Windhab E J 2004 Chem. Eng. Sci. $593045-58$

[5] Utada A S, Fernandez-Nieves A, Stone H A and Weitz D A 2007 Phys. Rev. Lett. 99094502

[6] Utada A S, Fernandez-Nieves A, Gordillo J M and Weitz D A 2008 Phys. Rev. Lett. 100014502

[7] Guillot P, Colin A, Utada A and Ajdari A 2007 Phys. Rev. Lett. 99104502

[8] Guillot P and Colin A 2008 Phys. Rev. E 78016307

[9] Huerre P and Monkewitz P A 1990 Ann. Rev. Fluid Mech. 22 473-537

[10] Herrada M, Gañán Calvo A and Guillot P 2008 Phys. Rev. E 78046312

[11] Moiré M, Peysson Y, Herzhaft B, Pannacci N, Gallaire F, Augello L, Dalmazzone C and Colin A 2017 Langmuir $332531-40$

[12] Chomaz J M 2005 Ann. Rev. Fluid Mech. 37 357-92

[13] Theofilis V 2011 Ann. Rev. Fluid Mech. 43 319-52

[14] Rubio-Rubio M, Sevilla A and Gordillo J M 2013 J. Fluid Mech. 729 471-83

[15] Gordillo J M, Sevilla A and Campo-Cortés F 2014 J. Fluid Mech. 738 335-57

[16] Tammisola O, Lundell F and Söderberg L D 2012 J. Fluid Mech. 713 632-58

[17] Cruz-Mazo F, Herrada M A, Ganan-Calvo M and Montanero J M 2017 J. Fluid Mech. 832 329-44

[18] Olsson E and Kreiss G 2005 J. Comput. Phys. 210 225-46

[19] Olsson E, Kreiss G and Zahedi S 2007 J. Comput. Phys. $225785-807$

[20] Hughes T J and Mallet M 1986 Comput. Methods Appl. Mech. Eng. 58 305-28

[21] Schenk O, Bollhöfer M and Römer R A 2008 SIAM Rev. 50 91-112

[22] Pozrikidis C 1992 Boundary Integral and Singularity Methods for Linearized Viscous Flow (Cambridge: Cambridge University Press)

[23] Popinet S 2003 J. Comput. Phys 190 572-600

[24] Prosperetti A and Tryggvason G 2007 Computational Methods for Multiphase Flow (Cambridge: Cambridge University Press)

[25] Brezzi F and Falk R S 1991 SIAM J. Numer. Anal. 28 581-90

[26] Hecht F 2012 J. Numer. Math. 20 251-65

[27] Hernandez V, Roman J E and Vidal V 2005 ACM Trans. Math. Softw. 31 351-62 
[28] Huerre P and Rossi M 1998 Hydrodynamic instabilities in open flows Hydrodynamics and Nonlinear Instabilities (ed C Godrèche and P Manneville) (Cambridge: Cambridge University Press)
[29] Heaton C J, Nichols J W and Schmid P J 2009 J. Fluid Mech. 629139

[30] Cordero M-L, Gallaire F and Baroud C N 2011 Phys. Fluids 23094111 